Supporting information

\title{
In-situ Monitoring of Electronic Structure in Modal Strong Coupling Electrode under Enhanced Plasmonic Water Oxidation
}

Yuchun Wang ${ }^{\dagger}$, Hiro Minamimoto ${ }^{\dagger}$, Ruifeng Zhou ${ }^{\dagger \ddagger}$, and Kei Murakoshi $\mathrm{i}^{\dagger *}$

$\dagger$ Department of Chemistry, Faculty of Science, Hokkaido University,

‡Institute for the Advancement of Higher Education, Hokkaido University. 


\section{Verification of other ATA electrodes}

In order to confirm the effect of the modal coupling, we further prepared additional two electrodes (NSC-ATA2, and NSC-ATA3), as in Fig. S1. One electrode (NSCATA2) shows the two maximum resonance peaks while other electrodes (NSC-ATA3) only appears the single peak. Theoretically, the condition for the definition of the strong coupling condition could be described as follow: ${ }^{\mathrm{S} 1}$

$$
\hbar \Omega>\sqrt{\frac{\gamma_{\mathrm{UB}}^{2}}{2}+\frac{\gamma_{\mathrm{LB}}^{2}}{2}}=\sqrt{\frac{\gamma_{\text {Cavity }}^{2}}{2}+\frac{\gamma_{\gamma_{\text {SSPR }}^{2}}^{2}}{2}}
$$

where $\hbar \Omega, \gamma_{\mathrm{UB}}$, and $\gamma_{\mathrm{LB}}$, represent the splitting energy defined as the difference of two peak positions, the linewidths of the upper and lower branch modes, respectively and $\gamma_{\text {cavity }}$ and $\gamma_{\text {LSPR }}$ are the linewidths of the nanocavity and LSPR modes, respectively. For SC-ATA described in the main text, $\hbar \Omega$ was $0.38 \mathrm{eV}$ while $\gamma_{\mathrm{UB}}$ and $\gamma_{\mathrm{LB}}$ were 0.35 and $0.22 \mathrm{eV}$, respectively. Therefore, $\sqrt{\frac{\gamma_{\mathrm{UB}}^{2}}{2}+\frac{\gamma_{\mathrm{LB}}^{2}}{2}}$ was calculated as $0.29 \mathrm{eV}$, indicating that the strong coupling condition could be satisfied. For the NSC-ATA2 shown as the gray spectrum in Fig. $\mathrm{S} 1, \hbar \Omega$ was $0.39 \mathrm{eV}, \gamma_{\mathrm{UB}}$ and $\gamma_{\mathrm{LB}}$ were 0.33 and $0.54 \mathrm{eV}$, respectively. The estimated $\sqrt{\frac{\gamma_{\mathrm{UB}}^{2}}{2}+\frac{\gamma_{\mathrm{LB}}^{2}}{2}}$ was about $0.45 \mathrm{eV}$. From these values, it could conclude that the strong coupling was not formed on NSC-ATA2, even showing comparable broad spectrum as that of SC-ATA (Figure 1). Considering the importance of the inlaid depth of Au nanoparticles for the formation of the strong coupling, the slight inhomogeneous structure of $\mathrm{TiO}_{2}$ which is derived from the atomic layer deposition method would result in such optical property difference as for the NSC-ATA2. Therefore, we named these two electrodes as NSC-ATA2 and NSC-ATA3. 


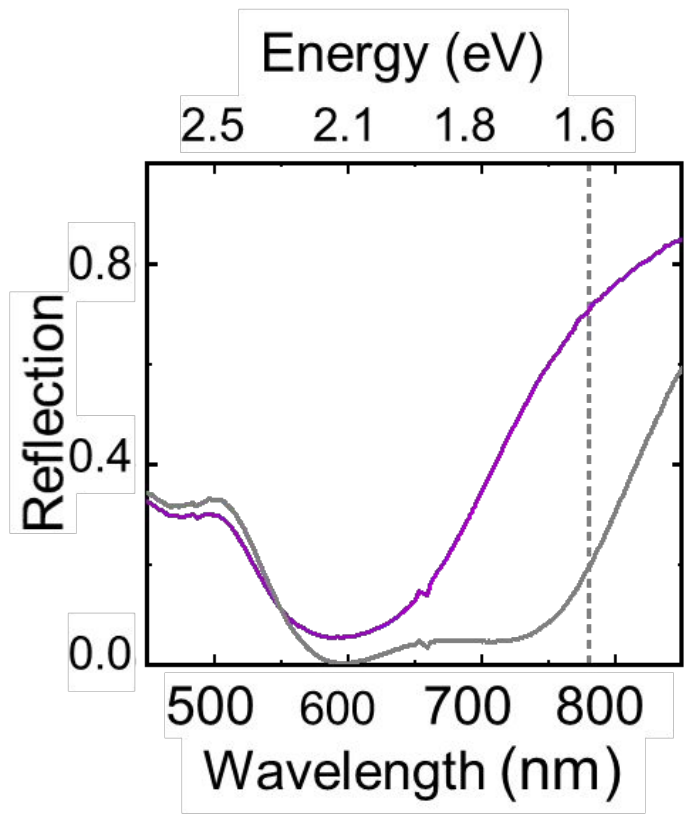

Figure S1. Reflection spectra of NSC-ATA2 and NSC-ATA3. Gray line indicates NSC-ATA2 and purple line indicated NSC-ATA3.

\section{Cross sectional view of ATA}

The cross section TEM image of ATA electrode is shown in Fig. S2. From this figure, the heterogeneous structure of $\mathrm{Au}$ nanoparticles and $\mathrm{TiO}_{2}$ on $\mathrm{Au}$ film was confirmed. The heterogeneity of $\mathrm{TiO}_{2}$ can be observed as the grains in $\mathrm{TiO}_{2}$ film. Amorphous carbon deposited on ATA structure is for the supporting material at TEM measurements.

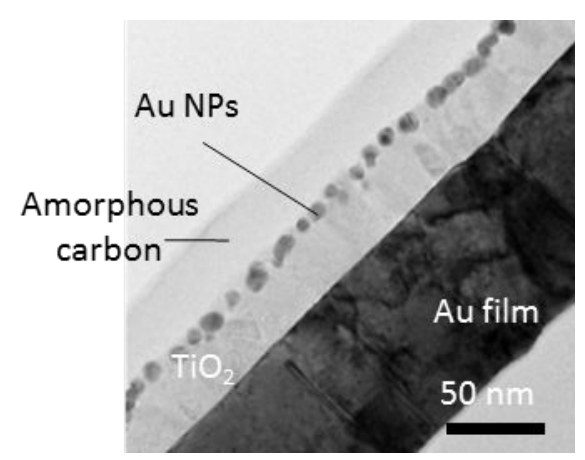

Figure S2. Cross sectional view of ATA. 


\section{Photocurrent measurements of each ATA electrode}

As mentioned in the main text, we have conducted photocurrent measurements using these ATA electrodes. The typical data of photocurrents for SC-ATA, SC-ATA2, NSCATA, NSC-ATA2 and NSC-ATA3 were obtained at $0.4 \mathrm{~V}$ as indicated in Fig.S3. It should be emphasized here that the generated photocurrent values were quite different at these electrodes. The spike features at NSC-ATA electrodes indicate insufficient charge separation efficiency of NSC-ATA2 electrode. ${ }^{\mathrm{S} 2}$

(a)

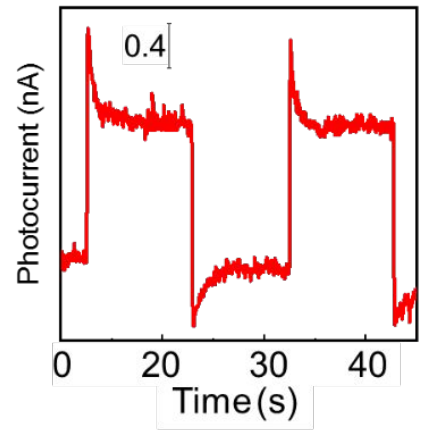

(d)

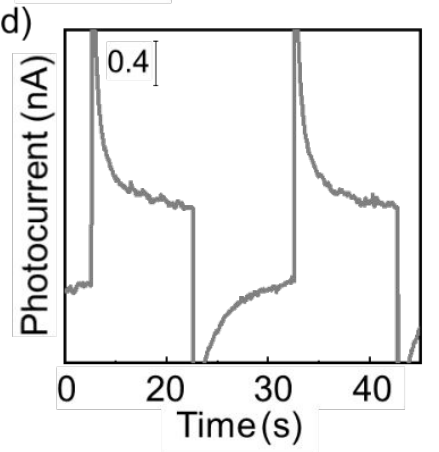

(b)

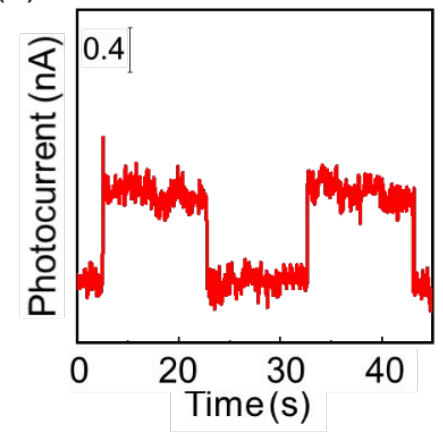

(c)

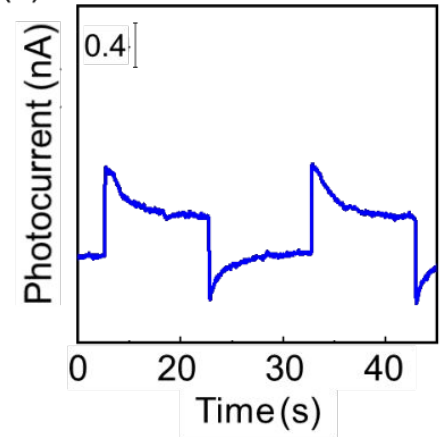

(e)

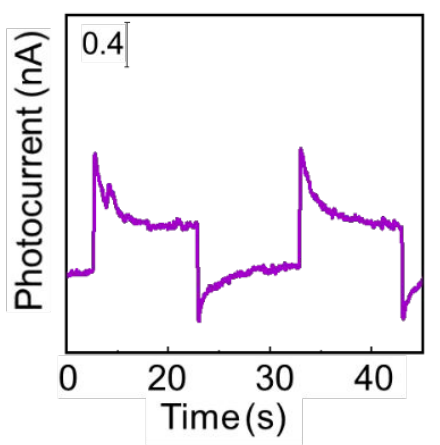

Figure S3. Photocurrents for (a) SC-ATA, (b) SC-ATA2, (c) NSC-ATA (d) NSCATA2 and (e) NSC-ATA3 at $0.4 \mathrm{~V}$.

The photocurrents for SC-ATA shows the quite stable photocurrent generations at all potentials as in the main text. For NSC-ATA2 the increasing value is very obvious (from about 0 to $0.6 \mathrm{nA}$ ). For NSC-ATA3, the photocurrent increased from 0.3 to 0.5 
nA during the potential scan. Relatively high efficient electron-hole separation results in photocurrent plateau at the positive electrochemical potential polarization than the flat band potential of n-type semiconductor electrodes. It is also important that the apparent spike features on NSC-ATA2 reflects the relatively lower charge separation efficiency compared to that on SC-ATA system. Therefore, these results prove that the charge separation efficiency for SC-ATA system would be quite higher compared to that for NSC-ATA.

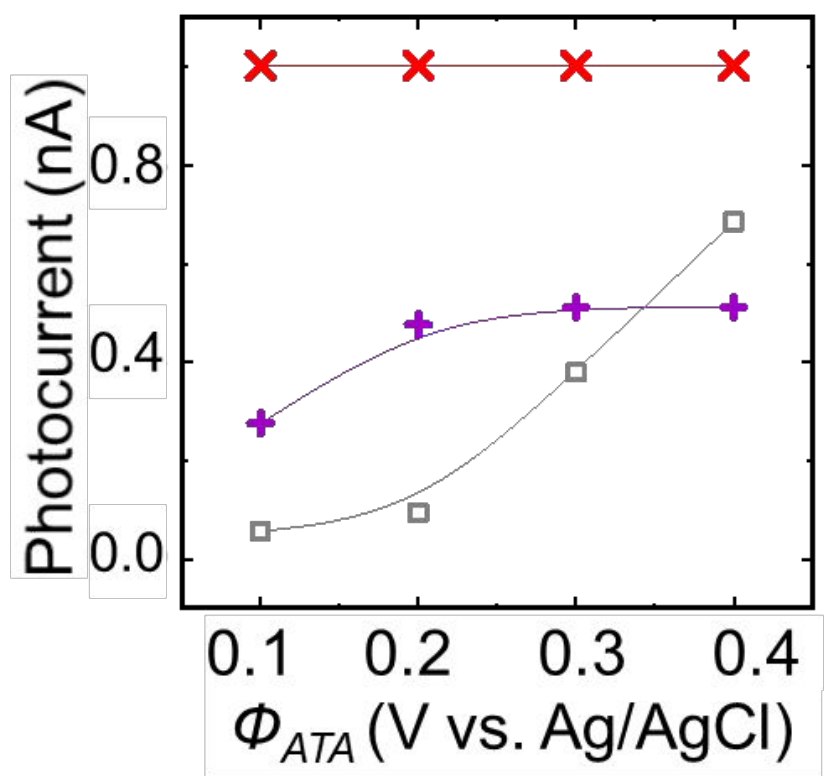

Figure S4. Relationship between photocurrents and applied potential. Red cross: SCATA2; purple cross: NSC-ATA2; gray hollow square: NSC-ATA3.

\section{Electrochemical in situ SERS of Gr / ATA}

Electrochemical SERS (EC-SERS) spectra collected using Gr/Au/ITO are shown in Fig. S5. The peak wavenumber was determined using the Lorentzian function as 
indicated as the broken line. All wavenumber values in the present paper were determined as the same way.
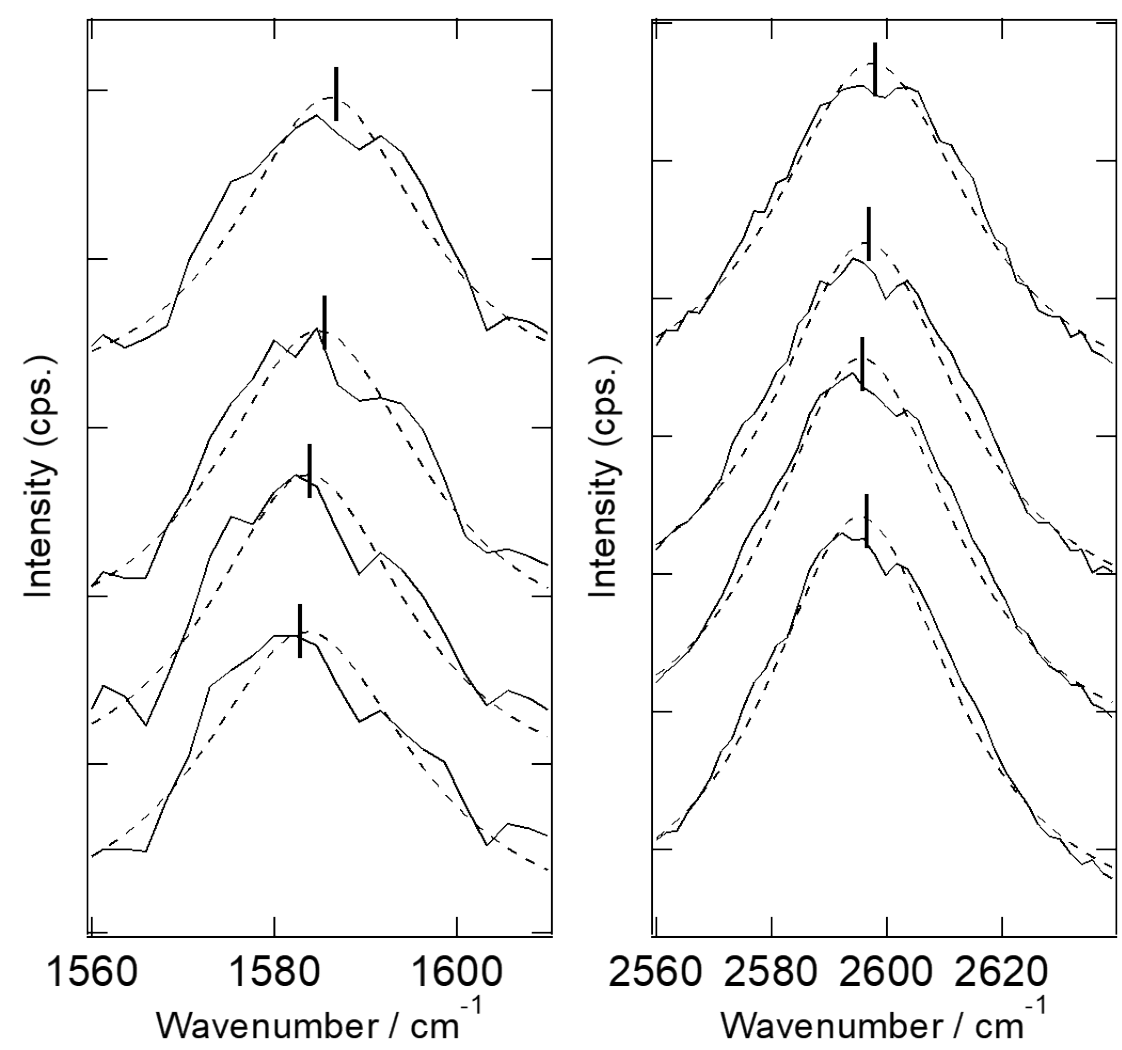

Figure S5. EC-SERS of Gr/Au/ITO obtained from $0.1 \mathrm{~V}$ (bottom) to $0.4 \mathrm{~V}$ (upper). The solid and broken lines are corresponding to raw and fitting data.

EC-SERS spectra of Gr / ATA on different electrodes are showing in Fig. S6. For all cases, D band $\left(\sim 1300 \mathrm{~cm}^{-1}\right)$ could be observed because of the plasmon effect. At about $1580 \mathrm{~cm}^{-1}$, G band appears and at about $2600 \mathrm{~cm}^{-1}, 2 \mathrm{D}$ band could be observed. For SC-ATA2, the position and intensity of $\mathrm{G}$ and 2D bands do not change obviously depending on the applied potential scan. This indicates that the shift of Fermi level is not associated with the change in the applied potential. However, when it comes to the 
cases of NSC-ATA2 and NSC-ATA3, both positions and intensities of G and 2D bands shift a lot, indicating the worse charge separation efficiency.
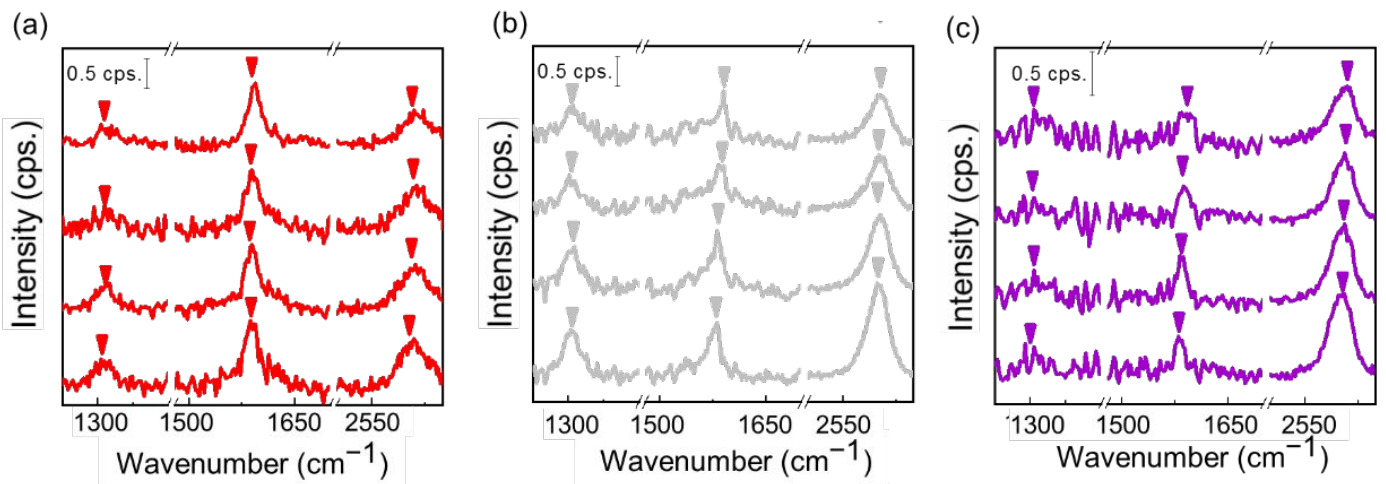

Figure S6. Raw EC-SERS of different sites at potential from $0.1 \mathrm{~V}$ (bottom) to 0.4 V(upper). (a) SC-ATA2. (b) NSC-ATA2. (c) NSC-ATA3. Triangles indicates the peak positions of $\mathrm{D}, \mathrm{G}$ and $2 \mathrm{D}$ peaks.

\section{Comparison of Gr / Au / ITO and Gr / ITO}

Figure S7a shows the potential-dependent $\omega_{\mathrm{G}}$ for Gr / Au / ITO and Gr / ITO. The value almost overlaps with each other, indicating that it was reasonable for us to use $\omega_{\mathrm{G}}$ of $\mathrm{Gr} / \mathrm{Au} / \mathrm{ITO}$ as a reference to calculate the Fermi level. Figure S7b presents the $I_{2 \mathrm{D}}$ / $I_{\mathrm{G}}$ of $\mathrm{Gr} / \mathrm{Au} / \mathrm{ITO}$ and Gr / ITO. Compared with Gr / ITO, the $I_{2 \mathrm{D}} / I_{\mathrm{G}}$ of $\mathrm{Gr} / \mathrm{Au} /$ ITO is much lower. This supports that plasmon excitation could change in the scattering rate. 

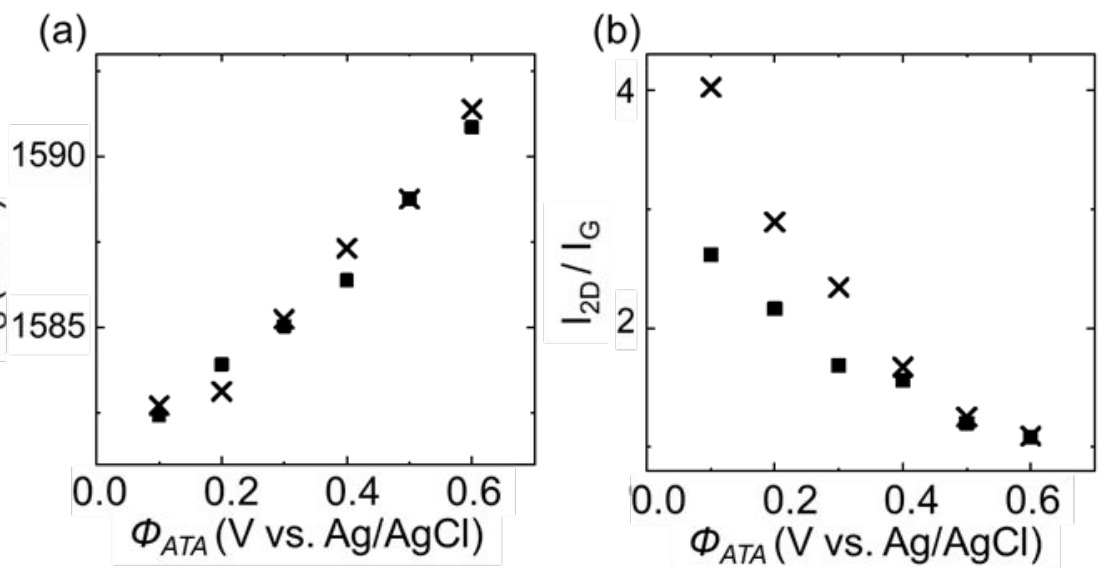

Figure S7. The $\omega_{\mathrm{G}}$ of $\mathrm{Gr} / \mathrm{Au} / \mathrm{ITO}$ and $\mathrm{Gr} / \mathrm{ITO}$ as the function of applied potential.

Black cross: Gr / ITO; black square: Gr / Au / ITO.

6. $\omega_{\mathrm{G}}$ and $\omega_{2 \mathrm{D}}$ as the function of applied potential 
The potential dependence of G and 2D bands for SC-ATA2, NSC-ATA2 and NSCATA3 are presented in Fig. S8. As for $\omega_{\mathrm{G}}$, it shifts when the potential changs positively

(a)

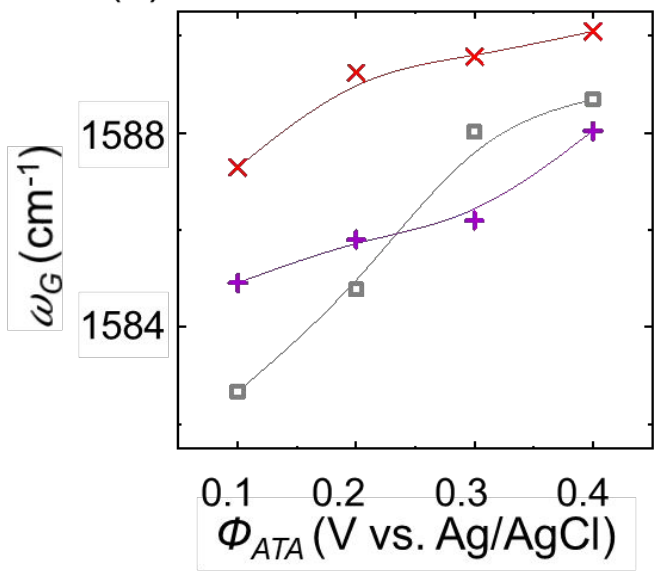

(b)

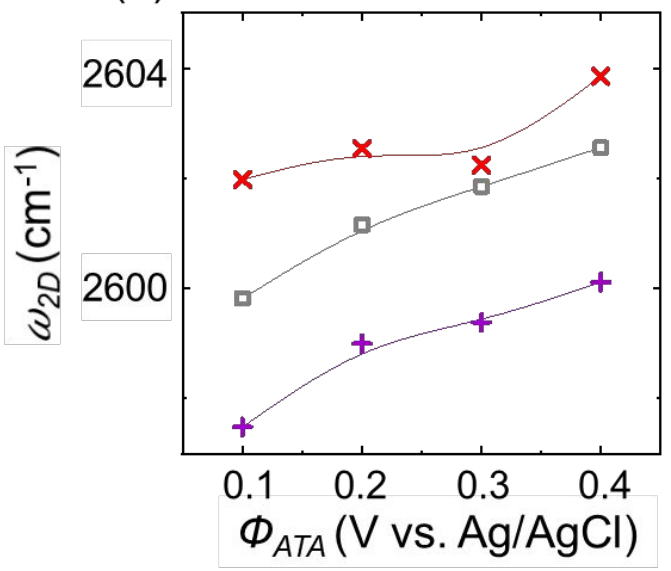

in all sites, but the extent of the blue shift is different. For SC-ATA2, it shifts from about 1587 to $1590 \mathrm{~cm}^{-1}$ with a variation of about $3 \mathrm{~cm}^{-1}$, while for NSC-ATA2, it exhibits the largest variation of about $8 \mathrm{~cm}^{-1}$. Since $\omega_{\mathrm{G}}$, represented the position of Fermi level, the variation with potential shift indicated that Fermi level also shifts when applied to different potential. In another word, the large variation of $\omega_{\mathrm{G}}$ means the lower charge separation efficiency. For $\omega_{2 \mathrm{D}}$, similar tendency occurs as for all sites it blue shifts during anodic potential scan. The value of $\omega_{2 \mathrm{D}}$ also indicates the interaction between graphene and substrate.

Figure S8. (a) $\mathrm{G}$ band position $\left(\omega_{\mathrm{G}}\right)$ and (b) $2 \mathrm{D}$ band position $\left(\omega_{2 \mathrm{D}}\right)$ at potential from $0.1 \mathrm{~V}$ to $0.4 \mathrm{~V}$. Red cross: SC-ATA2; purple cross: NSC-ATA2; gray hollow square: NSC-ATA3.

\section{REFERENCES}


S1. Shi, X.; Ueno, K.; Oshikiri, T.; Sun, Q.; Sasaki, K.; Misawa, H., Enhanced Water Splitting under Modal Strong Coupling Conditions. Nat. Nanotechnol. 2018, 13, $953-958$.

S2. Hagfeldt, A.; Lindström, H.; Södergren, S.; Lindquist, S. E., Photoelectrochemical Studies of Colloidal $\mathrm{TiO}_{2}$ Films: the Effect of Oxygen Studied by Photocurrent Transients. J. Electroanal. Chem. 1995, 381, 39-46. 